\title{
ON BISIMPLE SEMIGROUPS GENERATED BY A FINITE NUMBER OF IDEMPOTENTS
}

\author{
KARL BYLEEN \\ (Received 28 August 1981) \\ Communicated by T. E. Hall
}

\begin{abstract}
Non-completely simple bisimple semigroups $S$ which are generated by a finite number of idempotents are studied by means of Rees matrix semigroups over local submonoids $e S e, e=e^{2} \in S$. If under the natural partial order on the set $E_{s}$ of idempotents of such a semigroup $S$ the sets $\omega(e)=\left\{f \in E_{S}: f\right.$ $\leqslant e\}$ for each $e \in E_{S}$ are well-ordered, then $S$ is shown to contain a subsemigroup isomorphic to $S p_{4}$, the fundamental four-spiral semigroup. A non-completely simple bisimple semigroup is constructed which is gencrated by 5 idempotents but which does not contain a subsemigroup isomorphic to $S p_{4}$.
\end{abstract}

1980 Mathematics subject classification (Amer. Math. Soc.): 20 M 10.

\section{Introduction}

The generalization by D. Allen, Jr. [1] of the Rees theorem to a class of regular semigroups, and its improvement by D. B. McAlister [7] can be refined to obtain detailed information about the structure of bisimple semigroups which are generated by a finite number of idempotents. We use this approach to investigate an embedding question first raised in [3]: which non-completely simple bisimple idempotent-generated semigroups contain a subsemigroup isomorphic to $S p_{4}$ ?

The fundamental four-spiral semigroup $S p_{4}$ is presented by $\langle a, b, c, d| a=b a$, $a b=b=b c, c b=c=d c, c d=d=d a\rangle$ [3] and may be represented as the Rees matrix semigroup on $(\mathcal{E}(p, q)) ; 2,2 ;\left(\begin{array}{c}1 \\ 1 \\ 1\end{array}\right)$, over the bicyclic semigroup $e(p, q)$ [2]. It is an example of a non-completely simple bisimple idempotent generated semigroup which is the smallest such in the following sense: any $E$-chain linking

' Copyright Australian Mathematical Socicty 1982 
distinct comparable idempotents of a bisimple idempotent-generated semigroup

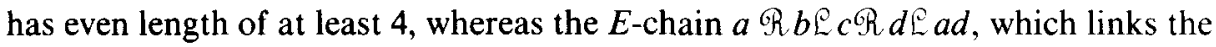
distinct comparable idempotents $a$ and $a d$ of $S p_{4}$, has length exactly 4 .

The embedding question asks for an analogue of the result that any non-completely simple bisimple regular semigroup contains a subsemigroup isomorphic to $\mathcal{C}(p, q)$. In [3] it was shown that any bisimple idempotent-generated semigroup $S$ in which $\omega(e)=\left\{f \in E_{S}: f \leqslant e\right\}$ is an $\omega$-chain for each $e \in E_{S}$ contains a subsemigroup isomorphic to $S p_{4}$. In [4] constructions of pseudosemilattices were used to provide examples of non-completely simple bisimple semigroups generated by infinitely many idempotents which fail to contain subsemigroups isomorphic to $S p_{4}$.

For each non-completely simple bisimple semigroup $S$ which is generated by a finite number of idempotents we shall construct in Section 2 a Rees matrix semigroup $\Re(e S e ; m, m ; P)$, also generated by a finite number of idempotents. which has $S$ as a homomorphic image. This Rees matrix cover will be used in Section 3 to show that if the sets $\omega(e)$ for $e \in E_{S}$ are well-ordered in such a semigroup $S$, then $S$ contains a subsemigroup isomorphic to $S p_{4}$. In Section 4 a Rees matrix semigroup over an inverse semigroup is constructed which is noncompletely simple, bisimple, is generated by 5 idempotents, but fails to contain a subsemigroup isomorphic to $S p_{4}$. The main results of this paper were announced at the Nebraska Conference on Semigroups, Lincoln, Nebraska in September 1980.

\section{A Rees matrix cover}

We first establish some results which will enable us to deduce properties of the Rees matrix cover. The submonoids $e S e, e \in E_{S}$, of a semigroup $S$ will be called local submonoids of $S$.

Proposition 2.1. Let $S$ be a regular semigroup, $e \in E_{S}$. If $S e S$ is finitely generated, then the local submonoid eSe is finitely generated.

Proof. Suppose we can prove the result in the special case $S=S e S$. Then the general result follows, for $S$ regular implies $S e S$ regular, and since $S e S=S e S \cdot e$

$\mathrm{SeS}$ is finitely generated, we conclude that $e \cdot S e S \cdot e=e S e$ is finitely generated.

So suppose $S=S e S$. Let $x_{1}, x_{2}, \ldots, x_{n}$ be generators for $S$. For each $x_{1}$ choose idempotents $u_{i}, v_{i}$ such that $v_{i} \mathfrak{R} x_{i} \mathfrak{R} u_{i}$, and elements $r_{i}, r_{i}^{\prime}, s_{i}, s_{i}^{\prime}$ such that $r_{i} r_{i}^{\prime}=u_{i}, r_{i}^{\prime} r_{i} \leqslant e, s_{i} s_{i}^{\prime}=v_{i}, s_{i}^{\prime} s_{i} \leqslant e$. Let $y=e\left(\prod_{l=1}^{w} x_{i}\right) e$ be an arbitrary element 
of $e S e$ where $1 \leqslant i_{1} \leqslant n, l=1,2, \ldots, w$. Then

$$
\begin{aligned}
y & =e\left(\prod_{l=1}^{w} u_{i_{l}} x_{i_{i}} v_{i_{l}}\right) e=e\left(\prod_{l=1}^{w} r_{i_{l}} r_{i}^{\prime} x_{i} s_{i} s_{i}^{\prime}\right) e \\
& =e r_{i_{1}}\left[\prod_{l=1}^{w-1}\left(r_{i}^{\prime} x_{i_{i}} s_{i}\right)\left(s_{i}^{\prime} r_{i_{l+1}}\right)\right]\left(r_{i_{w}}^{\prime} x_{i_{w}} s_{i_{w}}\right) s_{i_{w}}^{\prime} e .
\end{aligned}
$$

Thus the elements $e r_{i}, r_{i}^{\prime} x_{i} s_{i}, s_{i}^{\prime} r_{j}, s_{i}^{\prime} e, i, j=1,2, \ldots, n$, of $e S e$ generate $e S e$, so $e S e$ is generated by at most $n^{2}+3 n$ generators.

Let $L$ denote the 2-element lattice $L=\{0,1\}$ in which we write meet as $\cdot$, join as + , so that $0+0=0,0+1=1+0=1+1=1,0 \cdot 0=1 \cdot 0=0 \cdot 1=0$, $1 \cdot 1=1$. Thus under multiplication $L$ is the trivial group with 0 adjoined. The semigroup $L_{m}$ of all $m \times m$ matrices over $L, m$ a positive integer, may be interpreted as the semigroup of binary relations of an $m$-element set.

Proposition 2.2. Let $P$ be an $m \times m$ matrix over the lattice $L$ having diagonal entries all 1 and let $M$ denote the Rees matrix semigroup $\mathfrak{N}^{\circ}(1 ; m, m ; P)$ over the trivial group with 0 . Then $\Re^{\circ} \backslash\{0\}$ is generated by the $m$ idempotents $(i, 1, i)$, $i=1,2, \cdots, m$ if and only if all entries of $P^{r}$ equal 1 for some $r$.

Proof. The symbols $*$ and $\Sigma$ denote products in $\Re^{\circ}$. All other products are in $L_{m}$. Thus, for example $(i, 1, j) *(k, 1, l)=(i, 1, j) P(k, 1, l)$, where as usual $(i, 1, j)$ denotes the matrix with a 1 in the $(i, j)$ position, all other entries 0 . Suppose $A \in L_{m}$ has precisely one non-zero row, say row $i$, and that $B \in L_{m}$ has precisely one non-zero column, say column $j$. Then $A B$ has at most one non-zero entry, the $(i, j)$ entry. In view of the operations in $L$ there exists some $k$ such that (1) $A B=A(k, 1, k) B$. On the other hand, if, for some $k, A(k, 1, k) B \neq 0$, then (2) $A(k, 1, k) B=A B=(i, 1, j)$.

Suppose now that $\mathscr{R}^{\circ} \backslash\{0\}$ is generated by the $m$ idempotents $(i, 1, i)$. Then given $(i, 1, j) \in \pi^{\circ} \backslash\{0\}$ we can write

$$
(i, 1, j)=\prod_{l=1}^{n}\left(i_{l}, 1, i_{l}\right)=\left(i_{1}, 1, i_{1}\right) P\left(i_{2}, 1, i_{2}\right) P \cdots P\left(i_{w}, 1, i_{w}\right)
$$

which by repeated use of $(2)$ equals $\left(i_{1}, 1, i_{1}\right) P^{w-1}\left(i_{*}, 1, i_{w}\right)$. Therefore entry $(i, j)$ of $P^{w-1}$ is non-zero, so there exists $r$ such that all entries of $P^{r}$ equal 1.

Conversely, suppose that all entries of $P^{r}$ equal 1 for some $r$, and let $(i, 1, j) \in$ M ${ }^{\circ} \backslash\{0\}$. Then $(i, 1, i) P^{r}(j, 1, j)=(i, 1, j)$, so by repeated use of $(1)$ there exist $i=i_{1}, i_{2}, \ldots, i_{w}=j$ such that $\left(i_{1}, 1, i_{1}\right) P\left(i_{2}, 1, i_{2}\right) P \cdots P\left(i_{w}, 1, i_{w}\right)=(i, 1, j)$. Thus $(i, 1, j)=\prod_{l=1}^{\infty}\left(i_{l}, 1, i_{l}\right)$, so $\mathcal{M}^{\circ} \backslash\{0\}$ is generated by the $m$ idempotents $(i, 1, i)$. 
If $P=\left(p_{i j}\right)$ is an $m \times m$ matrix over a semigroup $S^{1}$ we define $\bar{P}=\left(\bar{p}_{i j}\right)$ to be the $m \times m$ matrix over the lattice $L=\{0,1\}$ such that

$$
\bar{p}_{i j}= \begin{cases}1 & \text { if } p_{i j}=1 \\ 0 & \text { if } p_{i j} \neq 1\end{cases}
$$

Proposition 2.3. Let $P$ be an $m \times m$ matrix over a semigroup $S^{1}$ with diagonal entries all 1 such that the entries of $\bar{P}^{r}$ are all 1 for some $r$. Then $\mathcal{M}\left(S^{1} ; m, m ; P\right)$ is generated by the $m$ idempotents $(i, 1, i)$ if and only if the entries of $P$ generate $S^{1}$.

Proof. Suppose $\mathscr{T}$ is generated by the $m$ idempotents $(i, 1, i)$ and let $s \in S^{1}$. Then $(1, s, 1)=\prod_{l=1}^{w}\left(i_{l}, 1, i_{l}\right)=\left(i_{1}, \prod_{l=1}^{w} p_{i_{1}, i_{l+1}}, i_{w}\right)$ for some $1 \leqslant i_{l} \leqslant m, l=$ $1,2, \ldots, w$, so $s$ is a product of entries of $P$.

Conversely, suppose that the entries of $P$ generate $S^{1}$. Let $(i, s, j) \in \mathfrak{O} R$. Then there exists $i_{l}, j_{l}, l=1,2, \ldots, w$ such that $s=\prod_{l=1}^{w} p_{i_{i}, j_{l}}$. Thus

$$
(i, s, j)=\left(i, 1, i_{1}\right)\left[\prod_{l=1}^{w-1}\left(i_{l}, 1, i_{l}\right)\left(j_{l}, 1, j_{l}\right)\left(j_{l}, 1, i_{l+1}\right)\right]\left(i_{w}, 1, i_{n}\right)\left(j_{n}, 1, j\right) \text {. }
$$

The partial function $\theta: \mathscr{M}^{\circ}(1 ; m, m ; \bar{P}) \rightarrow \mathscr{M}\left(S^{1} ; m, m ; P\right)$ defined by $(i, 1, j)$ $\rightarrow(i, 1, j), i, j=1,2, \ldots, m$, is a partial homomorphism in the sense that if $x, y, x y \in \mathscr{T}^{\circ} \backslash\{0\}$, then $(x \theta)(y \theta)=(x y) \theta$. Thus by Proposition 2.2 each of the factors in the product above is a product of the idempotents $(i, 1, i)$, $i=1,2, \ldots, m$. We conclude that $\mathscr{O}\left(S^{1} ; m, m ; P\right)$ is generated by these idempotents.

We observe that any matrix $P$ over a semigroup $S^{1}$ whose first row, first column, and diagonal entries are all 1 satisfies the hypothesis of Proposition 2.3, as does any matrix whose tridiagonal entries (those on the main diagonal and the two adjacent diagonals) are all 1.

The following result is a refinement of McAlister's Local Isomorphism Theorem [7], which in turn draws heavily on the ideas of D. Allen [1]. We denote the identity element $e$ of the local submonoid $e$ Se by 1 .

THEOREM 2.4. Let $S$ be a bisimple semigroup which is generated by a finite number of idempotents and let $e \in E_{S}$. Then $S$ is a homomorphic image by rectangular bands of a Rees matrix semigroup or (eSe; $m, m ; P)$ over the finitely. generated bisimple monoid eSe where

(1) On is generated by $m$ idempotents, $m$ a positive integer;

(2) the entries of $P$ generate $S$ Se and the tridiagonal entries of $P$ are all 1;

(3) the homomorphism is an isomorphism when restricted to any subsemigroup $\{i\} \times e S e \times\{j\}$. 
Proof. Let $S$ be a bisimple semigroup which is generated by a finite number of idempotents $x_{1}, x_{2}, \ldots, x_{n}$ and let $e \in E_{S}$. It is easy to check that the monoid $e S e$ is bisimple. By Proposition $2.1 e S e$ is finitely generated.

Since $S=\cup_{i=1}^{n} x_{i} S$ each $R$-class of $S$ is less than one of the finite number of maximal $\Re$-classes of $S$, and similarly for $\mathcal{L}$-classes. Since $S$ is bisimple and idempotent-generated the biordered set of $S$ is connected [3]. Thus there exists a sequence $e=e_{1}, e_{2}, e_{3}, \ldots, e_{m}$ of not necessarily distinct idempotents of $S$ with $e_{1} \Re e_{2} e_{3} \Re \cdots e_{m}$ such that each $x_{i}$ appears in the sequence. Let $I=$ $\left\{e_{1}, e_{2}, \ldots, e_{m}\right\}$. Then $I$ contains an idempotent from each maximal $\Re$-class and

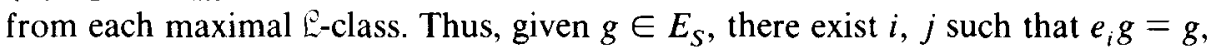
$g e_{j}=g$. To simplify notation in what follows we let $e_{0}=e=e_{1}$. For $i=$ $1,2, \ldots, m$ let

$$
\begin{gathered}
r_{i}= \begin{cases}e_{i} e_{i-2} \cdots e_{1} & \text { if } i \text { is odd } \\
e_{i-1} e_{i-3} \cdots e_{1} & \text { if } i \text { is even }\end{cases} \\
r_{i}^{\prime}= \begin{cases}e_{0} e_{2} \cdots e_{i-1} & \text { if } i \text { is odd } \\
e_{0} e_{2} \cdots e_{i} & \text { if } i \text { is even }\end{cases}
\end{gathered}
$$

The $m \times m$ matrix $P=\left(p_{i j}\right)$ is defined by $p_{i j}=r_{i}^{\prime} r_{j}$. Since $r_{i} r_{i}^{\prime}=e_{i}, r_{i}^{\prime} r_{i}=e$, $i=1,2, \ldots, m$, each $p_{i j}=\left(r_{i}^{\prime} r_{i}\right) r_{i}^{\prime} r_{j}\left(r_{j}^{\prime} r_{j}\right)$ belongs to $e S e$. Since $e=r_{i}^{\prime} r_{i+1}=r_{i+1}^{\prime} r_{i}$ for $i=1,2, \ldots, m-1$, the tridiagonal entries of $P$ are all 1 . Since each generator $x_{i}$ appears in $I$, any element of $e S e$ can be written in the form

$$
e\left(\prod_{l=1}^{n} e_{i_{l}}\right) e=e\left(\prod_{l=1}^{w} r_{i_{l}} r_{i_{l}}^{\prime}\right) e=r_{1}^{\prime} r_{i_{1}}\left(\prod_{l=1}^{w-1} r_{i_{l}}^{\prime} r_{i_{i+1}}\right) r_{i_{n}}^{\prime} r_{1} .
$$

Thus the entries of $P$ generate $e S e$.

Since $e S e$ is bisimple and regular it is easy to check that $\Re$ is also. By Proposition $2.3 \mathrm{O} \mathbb{R}$ is generated by the $m$ idempotents $(i, 1, i)$.

The mapping $\phi$ : o $(e S e ; m, m ; P) \rightarrow S$ by $(i, s, j) \rightarrow r_{i} s r_{j}^{\prime}$ is a homomorphism since $[(i, s, j)(k, t, l)] \phi=\left(i, s p_{j k} t, l\right) \phi$ and it maps or onto $S$ since any $s \in S$ can be written as

$$
s=s s^{\prime} s s^{\prime} s=r_{i} r_{i}^{\prime} s s^{\prime} \cdot s \cdot s^{\prime} s r_{j} r_{j}^{\prime}=r_{i}\left(r_{i}^{\prime} s r_{j}\right) r_{j}^{\prime}=\left(i, r_{i}^{\prime} s r_{j}, j\right) \phi
$$

for some $i, j$. To show that $\phi$ is an isomorphism into $S$ when restricted to the subsemigroup $\{i\} \times e S e \times\{j\}$ suppose that $(i, s, j) \phi=(i, t, j) \phi$. Then $r_{i} s r_{j}^{\prime}=$ $r_{i} t r_{j}^{\prime}$, so $r_{i}^{\prime} r_{i} s r_{j}^{\prime} r_{j}=r_{i}^{\prime} r_{i} t r_{j}^{\prime} r_{j}$ and thus ese $=e t e$ so $s=t$. To show that $\phi$ is a homomorphism by rectangular bands let $g \in E_{S}$ and suppose $(i, s, j) \phi=g$. Then $r_{i} s r_{j}^{\prime}=g$, so $r_{i} s r_{j}^{\prime} r_{i} s r_{j}^{\prime}=r_{i} s r_{j}^{\prime}$. Multiplying by $r_{i}^{\prime}$ on the left and $r_{j}$ on the right gives $s r_{j}^{\prime} r_{i} s=s$, so $(i, s, j)$ is idempotent. If $(k, t, l) \phi=g$, then $r_{k} t r_{i}^{\prime}=r_{i} s r_{j}^{\prime}$, so $(i, s, j)(k, t, l)(i, s, j)=\left(i, s r_{j}^{\prime} r_{k} t r_{l}^{\prime} r_{i} s, j\right)=(i, s, j)$, so $g \phi^{-1}$ is a rectangular band, as required. 


\section{Embedding $S p_{4}$ in certain bisimple idempotent-generated semigroups}

The defining relations for the fundamental four-spiral semigroup $S p_{4}$ imply that $a, b, c, d, a d$ are idempotents with $a d \leqslant a$. Although $a d<a$ in $S p_{4}, S p_{4}$ has a least non-identity congruence, and this congruence identifies $a d$ and $a$. Therefore a semigroup $S$ contains a subsemigroup isomorphic to $S p_{4}$ if and only if $S$ contains idempotents $a, b, c, d$ with $a \Re b \ell c \Re d$ such that $d a=d$ and $a d \neq a$.

Lemma 3.1. Let $S$ and $T$ be semigroups and let $\phi: S \rightarrow T$ be a homomorphism from $S$ onto $T$ which does not identify distinct comparable idempotents of $S$. If $S$ contains a subsemigroup isomorphic to $\mathrm{Sp}_{4}$, then so does $T$.

Proof. The restriction of $\phi$ to the subsemigroup of $S$ isomorphic to $S p_{4}$ must induce the identity congruence, since distinct comparable idempotents are not identified.

Lemma 3.2. Let $S$ be an inverse semigroup with natural partial order $\leqslant$. A Rees

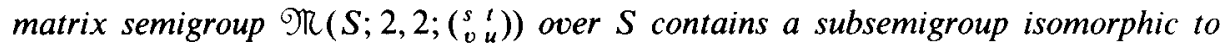
$\mathrm{Sp}_{4}$ if and only if there exist elements $a, b, c, d \in S$ such that

(1) $a \Re b\llcorner c \Re d$

(2) $a \leqslant s^{-1}, b \leqslant v^{-1}, c \leqslant u^{-1}, d \leqslant t^{-1}$; and

(3) either (i) $d s a=d$, atd $<a$ or (ii) $d s a<d$, atd $=a$.

Proof. Suppose that $\Re$ contains a subsemigroup isomorphic to $S p_{4}$. Since any $\mathcal{R}$-class or $\mathcal{E}$-class of $\Re$ contains at most one idempotent which belongs to a subsemigroup $\{i\} \times S \times\{j\}$, there exist idempotents $(1, a, 1) \mathscr{R}(1, b, 2) \mathcal{L}(2, c, 2) \mathscr{R}(2, d, 1)$ such that either (i) $(2, d, 1)(1, a, 1)=$ $(2, d, 1), \quad(1, a, 1)(2, d, 1)<(1, a, 1)$ or (ii) $(2, d, 1)(1, a, 1)<(2, d, 1)$, $(1, a, 1)(2, d, 1)=(1, a, 1)$. These conditions on idempotents of $\mathfrak{K}$ imply conditions (1), (2), (3) on the elements $a, b, c, d$ of $S$.

Conversely, suppose $a, b, c, d$ are elements of $S$ such that (1), (2) and (3) hold. Then $(1, a, 1),(1, b, 2),(2, c, 2),(2, d, 1)$ are idempotents of $\Re$ which generate a subsemigroup isomorphic to $S p_{4}$.

We follow the usual convention of calling the semilattice $E$ of idempotents of an inverse semigroup well-ordered if the reverse of the natural partial order on $E$ is a well-ordering of $E$. Below $\leqslant$ denotes the usual order on the ordinals.

THEOREM 3.3. Let $S$ be a non-completely simple bisimple semigroup which is generated by a finite number of idempotents. If $E_{e S e}$ is well-ordered for each $e \in E_{S}$, then $S$ contains a subsemigroup isomorphic to $S_{4}$. 
Proof. Suppose $S$ is a non-completely simple bisimple semigroup which is generated by a finite number of idempotents in which $E_{e S e}$ is well-ordered for each $e \in E_{S}$. Let $\Re(e S e ; m, m ; P)$ be a Rees matrix cover for $S$, as guaranteed by Theorem 2.4. To show that $S$ contains a subsemigroup isomorphic to $S p_{4}$ it suffices by Lemma 3.1 to show that $\mathfrak{N}$ does. The maximum idempotent-separating congruence $\mu$ on $e S e$ induces an idempotent-separating homomorphism from भT $(e S e ; m, m ; P)$ into $\Re\left(e S e / \mu ; m, m ; P \mu^{\natural}\right)$ where for $P=\left(p_{i j}\right)$ we denote by $P \mu^{\natural}$ the matrix $\left(p_{i}, \mu^{\natural}\right)$. Let $E=E_{e S e}$. The bisimple semigroup $e S e / \mu$ is isomorphic to a full inverse subsemigroup of $T_{E}$, which since $T_{E}$ is combinatorial ( $E$ is well-ordered) implies that $e S e / \mu$ is isomorphic to $T_{E}$. Thus $\Re\left(e S e / \mu ; m, m ; P \mu^{\natural}\right)$ is isomorphic to a Rees matrix semigroup $\Re\left(T_{E} ; m, m ; P^{\prime}\right)$ over $T_{E}$. Since $\mathscr{N}\left(T_{E} ; m, m ; P^{\prime}\right)$ is an idempotent-separating homomorphic image of $\Re(e S e ; m, m ; P)$, to prove the theorem it suffices to show that $\Re\left(T_{E} ; m, m ; P^{\prime}\right)$ contains a subsemigroup isomorphic to $S p_{4}$.

Since $E$ is uniform, $E$ is isomorphic to an ordinal power of $\omega[5]$, [10] say $E=\omega^{r}$, and since $S$ is non-completely simple, $r \geqslant 1$. Given ordinals $a, b<\omega^{r}, E a$ will denote the principal ideal $\left\{\mathrm{x}: \mathrm{a} \leqslant \mathrm{x}<\omega^{r}\right\}$ of $E$ generated by $a$, and the unique principal ideal isomorphism from $E a$ to $E b$ is given by $a+x \rightarrow b+x$ for $0 \leqslant x<\omega^{r}$ (usual addition of ordinals). Below we will use the fact, which follows from the normal form for ordinals [9], that for ordinals $x$ and $a, x=\omega^{a}+x \Rightarrow x$ $\geqslant \omega^{a+1}(*)$.

We claim that some entry of $P^{\prime}$ has no fixed point. Suppose to the contrary that each of the finitely many entries of $P^{\prime}$ has a fixed point, and let $\bar{x}$ be their supremum. Then each entry of $P^{\prime}$ is the identity on $\bar{X}=\left\{x: \bar{x} \leqslant x<\omega^{r}\right\}$. Thus, since the entries of $P^{\prime}$ generate $T_{E}$, each element of $T_{E}$ is the identity on $\bar{X}$. But this is impossible, since if $r$ is not a limit ordinal then the principal ideal isomorphism $E 0 \rightarrow E \omega^{r-1}$ has no fixed point by (*), while if $r$ is a limit ordinal, then $E 0 \rightarrow E \omega^{k}$, where $k$ is chosen so that $\bar{x}<\omega^{k}$, does not fix $w^{k}$ (again by $(*)$ ). This establishes the claim.

Of those entries of $P^{\prime}$ without fixed points let $\eta$ be one which is closest to the main diagonal of $P^{\prime}$. If $\eta$ appears above the diagonal it belongs to a $2 \times 2$ submatrix of $P^{\prime}$ of the form

\begin{tabular}{r|c|c|}
\multicolumn{1}{c}{} & \multicolumn{1}{c}{$i$} & $i+1$ \\
\cline { 2 - 3 }$j$ & $\alpha$ & $\eta$ \\
\cline { 2 - 3 }$j+1$ & $\beta$ & $\gamma$ \\
\cline { 2 - 3 } & &
\end{tabular}

where $\alpha, \beta, \gamma$ have fixed points (the case where $\eta$ lies below the diagonal is entirely similar). Thus there exist elements $u, v \in E$ which are fixed by $\alpha, \beta, \gamma$ 
such that $u \eta=v$. Since the principal ideal isomorphisms $E v \rightarrow E v, E v \rightarrow E v$, $E v \rightarrow E v, E v \rightarrow E u$ satisfy the conditions (1),(2),(3) on $a, b, c, d$ of Lemma 3.2, $\mathscr{N}\left(T_{E} ; m, m ; P^{\prime}\right)$ contains a subsemigroup isomorphic to $S p_{4}$, as required.

\section{The counterexample}

Motivated by the Rees matrix cover described in Theorem 2.4 we construct a semigroup which yields a negative answer to the following question, posed as problem B2 in [8]: does every non-completely simple bisimple semigroup which is generated by a finite number of idempotents contain a subsemigroup isomorphic to $S p_{4}$, the fundamental four-spiral semigroup?

EXAmple 4.1. Let $S$ denote the $P$-semigroup $P(G, \mathcal{X}$, O $)$ [6] where $G=\mathbf{Z} \times \mathbf{Z}$ is the direct product of two copies of the group of integers under addition, $\mathscr{X}=\mathbf{Z} \times \mathbf{Z}$ is the direct product of two copies of the semilattice of integers under the usual order, and $\mathcal{Y}=\mathbf{Z}^{-} \times \mathbf{Z}^{-}$is the subsemilattice and ideal of $\mathscr{X}$ consisting of all elements of $\mathscr{X}$ whose components are both $\leqslant 0$. Let $G$ act on $\mathscr{X}$ by order automorphisms as follows: if $g=(e, f), A=(m, n)$, then $g A=(e+m, f+n)$. Under the multiplication $(A, g) \cdot(B, h)=(A \wedge g B, g h), S=\left\{(A, g) \in g_{\mathcal{G}} \times\right.$ $\left.G: g^{-1} A \in \mathcal{Y}\right\}$ becomes an $E$-unitary inverse semigroup with semilattice $\mathcal{O}$ and maximum group homomorphic image $S / \sigma \cong G$. The natural partial order on $S$ is given by $(A, g) \leqslant(B, h)$ if and only if $A \leqslant B$ and $g=h$. We will denote the element $(A, g)$ in $S$, where $A=(m, n), g=(e, f)$ by $(m, n ; e, f)$.

Let $p=(0,0 ; 1,0), r=(-1,0 ;-1,1)$. Then $p^{-1}=(0,0 ;-1,0), r^{-1}=(0,-1 ; 1$, $-1)$ and $S$ is generated as a semigroup by $p, r, p^{-1}, r^{-1}$. Let $\mathscr{N}(S ; 5,5 ; P)$ be the Rees matrix semigroup over $S$ with matrix

$$
P=\left(\begin{array}{lllll}
1 & 1 & r & r & p \\
1 & 1 & 1 & r & r \\
r^{-1} & 1 & 1 & 1 & r \\
r^{-1} & r^{-1} & 1 & 1 & 1 \\
p^{-1} & r^{-1} & r^{-1} & 1 & 1
\end{array}\right)
$$

As usual, 1 denotes the identity element of $S$, so $1=(0,0 ; 0,0)$. By Proposition $2.3 \Re$ is generated by the 5 idempotents $(i, 1, i), i=1,2,3,4,5$. Since $S$ is bisimple but not completely simple, the same is true of $\mathfrak{T}$.

We claim that $\mathscr{T}$ does not contain a subsemigroup isomorphic to $S p_{4}$. Suppose to the contrary that $\mathfrak{R}$ does. Then there exist $i, j, k, l$ such that the subsemigroup 
$\{i, j\} \times S \times\{k, l\}$ of $\mathscr{T}$ contains a subsemigroup isomorphic to $S p_{4}$. Thus there exists a submatrix $P^{\prime}=\left(\begin{array}{ll}s & t \\ v & u\end{array}\right)$ of $P$ and elements $a=(A, g), b=(B, h), c=(C, k)$, $d=(D, l)$ of $S$ satisfying (1), (2), (3) of Lemma 3.2.

The $R$ and $Q$ relations of (1) imply that $A=B, h^{-1} B=k^{-1} C$, and $C=D$, hence $A=h k^{-1} D$. If (3)(i) holds, then $d s a=d$ so $d a^{-1} a=d$ and thus $d^{-1} d \leqslant a^{-1} a$. But since also atd $<a$, and thus $a d^{-1} d<a$, we conclude $d^{-1} d<a^{-1} a$, so $l^{-1} D<g^{-1} A$ and thus $D<l g^{-1} h k^{-1} D$. If (3)(ii) holds, then similarly we obtain $\lg ^{-1} h k^{-1} D<D$. Let $\alpha=l g^{-1} h k^{-1}$. Then in either case $D$ and $\alpha D$ are distinct and comparable. By (2), $\alpha=\left(t^{-1} s v^{-1} u\right) \sigma^{\natural}$.

Let $s \sigma^{\natural}=\left(w_{1}, w_{2}\right), t \sigma^{\natural}=\left(x_{1}, x_{2}\right), u \sigma^{\natural}=\left(y_{1}, y_{2}\right), v \sigma^{\natural}=\left(z_{1}, z_{2}\right)$. Then

$$
P^{\prime} \sigma^{\natural}=\left(\begin{array}{ll}
s \sigma^{\natural} & t \sigma^{\natural} \\
v \sigma^{\natural} & u \sigma^{\natural}
\end{array}\right)=\left(\left(\begin{array}{ll}
w_{1} & x_{1} \\
z_{1} & y_{1}
\end{array}\right),\left(\begin{array}{ll}
w_{2} & x_{2} \\
z_{2} & y_{2}
\end{array}\right)\right)
$$

and $\alpha=\left(w_{1}-x_{1}+y_{1}-z_{1}, w_{2}-x_{2}+y_{2}-z_{2}\right)$. Since $D$ and $\alpha D$ are distinct and comparable, the components of $\alpha$ are not both zero and either both are $\geqslant 0$ or both are $\leqslant 0$. We will be helpful to call the quantity $w-x+y-z$ associated with the $2 \times 2$ matrix $W=\left(\begin{array}{cc}w & x \\ z & y\end{array}\right)$ of integers the increment of $W$. Let

$$
P_{1}=\left(\begin{array}{rrrrr}
0 & 0 & -1 & -1 & 1 \\
0 & 0 & 0 & -1 & -1 \\
1 & 0 & 0 & 0 & -1 \\
1 & 1 & 0 & 0 & 0 \\
-1 & 1 & 1 & 0 & 0
\end{array}\right) \quad \text { and } \quad P_{2}=\left(\begin{array}{rrrrr}
0 & 0 & 1 & 1 & 0 \\
0 & 0 & 0 & 1 & 1 \\
-1 & 0 & 0 & 0 & 1 \\
-1 & -1 & 0 & 0 & 0 \\
0 & -1 & -1 & 0 & 0
\end{array}\right)
$$

be the matrices of first and second components, respectively, of the elements of $P^{\natural}$. To obtain the contradiction it suffices to show that if a $2 \times 2$ submatrix of $P_{1}$ has positive (negative) increment, then the corresponding $2 \times 2$ submatrix of $P_{2}$ has negative (positive) increment. This is clear for any pair of corresponding $2 \times 2$ submatrices which do not contain the $(1,5)$ or $(5,1)$ positions, for then the increments are negatives of each other. It is true by default for the pair of $2 \times 2$ submatrices consisting of the corner entries (increments both 0 ) and is easily checked for the $2 \times 2$ submatrices in the upper right and lower left corners. Any other pair of corresponding $2 \times 2$ submatrices must contain exactly one of the positions $(1,5),(5,1)$, we may assume $(1,5)$ by symmetry, so has the form

$$
\left(\begin{array}{cc}
w & 1 \\
z & y
\end{array}\right)\left(\begin{array}{cc}
-w & 0 \\
-z & -y
\end{array}\right)
$$

where $w \leqslant 0, y \leqslant 0, z \geqslant 0$. The increment of the first is $\leqslant 0$, that of the second is $\geqslant 0$. This contradicts the existence of $\alpha$, and forces us to conclude that $\mathfrak{N}$ contains no subsemigroup isomorphic to $S p_{4}$. 


\section{References}

[1] D. Allen, Jr., 'A generalization of the Rees theorem to a class of regular semigroups', Semigroup Forum 2 (1971), 321-331.

[2] K. Byleen, 'Regular four-spiral semigroups, idempotent-generated semigroups and the Rees construction', Semigroup Forum 22 (1981), 97-100.

[3] K. Byleen, J. Meakin and F. Pastijn, 'The fundamental four-spiral semigroup', J. Algebra 54 (1978), 6-26.

[4] K. Byleen, J. Meakin and F. Pastijn, 'Building bisimple idempotent-generated semigroups', $J$. Algebra 65 (1980), 60-83.

[5] J. W. Hogan, 'Bisimple semigroups with idempotents well-ordered'. Semigroup Forum 6 (1973). 298-316.

[6] D. B. McAlister, 'Groups, semilattices and inverse semigroups'. Trans. Amer. Math. Soc. 192 (1974), 227-244.

[7] D. B. McAlister, 'Regular Rees matrix semigroups and regular Dubreil-Jacotin semigroups'. J. Austral. Math. Soc., Ser A 31 (1981), 325-336.

[8] D. B. McAlister and R. McFadden (editors), Proceedings of the Symposium on Regular Semigroups, (Northern Illinois University, DeKalb, April 1979).

[9] W. Sierpinski, Cardinal and ordinal numbers, Second edition revised (PWN-Polish Scientific Publishers, Warszawa, 1965).

[10] G. White, 'The dual ordinal of a bisimple inverse semigroup', Semigroup Forum 6 (1973). 295-297.

Department of Mathematics, Statistics, and Computer Science

Marquette University

Milwaukee, Wisconsin 53233

U.S.A. 